\title{
Repetition in Pirandello's Prose and Translation into Albanian Language
}

\author{
Prof. As. Dr. Mirela Papa \\ Docente di Traduzione e Interpretariato, Università di Tirana \\ papamirela@yahoo.com \\ Dr. Alva Dani \\ Docente di Letteratura Italiana, Università di Scutari \\ alvadani2001@yahoo.it
}

Doi:10.5901/jesr.2014.v4n4p318

\begin{abstract}
Based on an analysis of Pirandello's prose, it is noticed that the language used by him is a common one, an anti-literary one, anti-academic and its basis is the spoken language. Pirandello uses the spoken language as means to achieve his aims in order to create a realistic and drilling language. Pirandello is interested in these aspects of everyday spoken Italian that best express the inner world of his characters, who turn into spokespersons of his ideas. Among the characteristics of spoken language is the repetition, which constitutes one of the most common figures of speech applied by Pirandello. Text Linguistics studies regard this figure of speech as one of the syntactic and semantic relations on which depends the cohesion of the discourse. The effects presumed via word repetition, consciously and in a well-processed manner, or even unconsciously constitute the object of psychological and linguistic analysis. The goals of Pirandello applying repetition are many. In this paper there are exactly the repetitions used by Pirandello that will serve for the analysis, The strategies followed by translators aiming at translating this figure into Albanian language texts will be the object of analysis. Whether or not this unique feature is preserved for the Albanian reader is to be reviewed.
\end{abstract}

Keywords: figures of speech, literature, prose, repetition, translation.

\section{Introduzione Allo Studio}

Lo scrittore italiano Luigi Pirandello ha un suo stile particolare nella scrittura. Quando scrive lo fa con la spontaneità di una parlata tra amici. Il suo modo di scrittura si avvicina di più alla lingua parlata. Gli interessano soprattutto quegli aspetti dell'italiano familiare parlato che esprimono meglio il mondo interiore dei suoi personaggi. Tra le caratteristiche della lingua parlata è anche la ripetizione, una figura del discorso molto usata da Pirandello. In questo studio si prenderanno in analisi le ripetizioni che usa Pirandello nelle sue novelle e si vedrà se i traduttori albanesi hanno riportato o meno nei testi tradotti questa caratteristica del linguaggio di Pirandello.

\section{Caratteristiche della Lingua delle Novelle di Pirandello}

Lo stesso Pirandello diceva che di stili l'artista non ne ha uno solo, ma tanti quanti sono i momenti della sua creazione, uno per ciascuno. Questa dichiarata esigenza di individualità esprime chiaramente l'atteggiamento antiletterario del professor Pirandello (Puglisi, 1968: 134). «Se letteratura, o meglio, tradizione letteraria ha mai fatto impedimento al libero sviluppo d'una lingua, questa più d'ogni altra è l'italiana» scrive ancora nei Saggi, poesie e scritti vari criticando la retorica. Ecco perché la lingua di Pirandello è antiletteraria, spontanea. Nel suo linguaggio si nota «la tendenza, prima di tutto e soprattutto, al dialogo, la preponderanza ch'esso via via va assumendo nel corpo della o delle opere. L'elemento narrativo........) si rimpicciolirà per cedere il posto al discorso diretto, vivo, saltellante. Pirandello ha bisogno di parlare, di scrivere come se parlasse, di rivolgersi, allorché si accinge a raccontare un fatto della vita, per lo più inesplorato sino allora, a una seconda persona, di proiettare, come di solito ci accade quando siamo soli e parliamo con noi stessi... » (Puglisi, 1968: 135) Vediamo dunque in Pirandello «l'aspirazione evidente verso un lessico discorsivo e prosastico» (Orilia, 1967: 711). Nel suo stile narrativo, espressivo e senza retorica, la prosa italiana incontra un modello da seguire. Pirandello usa la lingua parlata come uno strumento per realizzare i suoi scopi per creare «un linguaggio pungente e realistico, senza indugi oziosi e blandi compiacimenti linguistici» (Munafò citato da Navarro Blanco, 1990: 100). Sono pochi gli studi concentrati sul lessico delle novelle di Pirandello e sulla provenienza di tale lessico. Pirandello come lo dimostrano i suoi scritti (Lo Vecchio-Musti 1960) sente una grande preoccupazione per la lingua italiana della sua epoca 
e cercherà di fare uso di tutti gli strati della lingua senza rifiutare le parole per la loro provenienza. II suo è il lessico appartenente alla lingua comune impiegata dagli italiani dell'epoca.

\section{Le Ripetizioni}

Una delle figure retoriche più impiegate da Pirandello è, senza ombra di dubbio, la ripetizione. Gli studi attuali della linguistica del testo la considerano una delle relazioni sintattiche e semantiche dalle quali dipende la coesione del discorso. Considerata una delle costanti del discorso poetico, la sua esistenza è condizionata dalle rime, assonanze, allitterazioni, cadenze ritmiche, ecc. (Navarro Blanco, 1990: 115). Gli effetti che si possono ottenere tramite la ripetizione delle parole in modo conscio o incoscio sono oggetto di analisi linguistica e psicologica. Gli scopi per cui Pirandello usa la ripetizione sono molteplici: insistere su un'idea precedentemente formulata oppure conseguire un grado maggiore di intensità, solennità e ricchezza di espressione. (Navarro Blanco, 1990: 115)

Vediamo alcuni esempi di ripetizioni in alcune delle novelle di Pirandello:

\subsection{Ripetizioni di verbi}

$>$ E quel boja di vetturino intanto aveva il coraggio di dire che bisognava saperla guidare, lasciarla andare col suo verso, perché ombrava, ombrava e, a frustarla, ritta gli si levava come una lepre, certe volte, quella bestiaccia lì. (Le sorprese della scienza)

- Dhe ai karrocieri xhahil, ndërkaq, kishte guxim e thoshte se duhej të dije ta ngisje kalin, duhej ta lije të ecte për qejf të tij, sepse trembej, trembej edhe po t'i bije me kamxhik; nganjëherë ajo kafshë e mallkuar ngrihej arithi, thikë përpjetë, bash si lepur. (Befasitë e shkencës, p. 100, "Tregime e novela", Dituria, Tiranë 1999)

$>$ Andate, andate, - gli ordinò. - Dite così che... se posso... tra poco... Ora andate, andate! (Come gemelle)

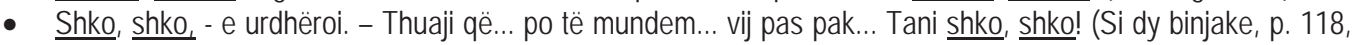
«Zoja prej dylli», Toena, Tiranë 2008)

$>$ Ma il letto tenta, sa! Tenta, tenta. (Niente)

- Por shtrati të tërheq, e dini? Të tërheq, të tërheq. (Hiç asgjë, p. 83, «Zoja prej dylli», Toena, Tiranë 2008)

$>$ Dormo dove posso. Mangio quando posso. Vesto come posso. (Niente)

- Fle ku mundem. Ha kur mundem. Vishem si mundem. (Hiç asgjë, p. 83, «Zoja prej dylli», Toena, Tiranë 2008)

$>$ Se ne può andare! Se ne può andare! (Niente)

- Mund të shkoni! Mund të shkoni! (Hiç asgjë, p. 86, «Zoja prej dylli», Toena, Tiranë 2008)

$>$... sempre e da per tutto, non aveva fatto altro che leggere, leggere, leggere. (Mondo di carta)

- Gjithmonë dhe kurdoherë vetëm se kishte lexuar, lexuar, lexuar. (Botë letre, p. 62, «Zoja prej dylli», Toena, Tiranë 2008)

> Lei mi vuole rovinare! Se ne vada! Se ne vada! Non può più stare qua! Mi lasci solo! Se ne vada! (Mondo di carta)

- Ju nuk ma doni të mirën! Largohuni! Largohuni! Nuk mund të rrini këtu! Më lini vetëm! Largohuni! (Botë letre, p. 67, «Zoja prej dylli», Toena, Tiranë 2008)

$>$...le erano rimasti appena gli occhi per piangere: piangere sangue! ecco, sangue! (La fede)

- ...i kishin mbetur vetëm sytë për të vajtuar, për të qarë me lot gjaku! (Besimi, p. 54, «Zoja prej dylli», Toena, Tiranë 2008)

> Era abituata a volare, lei, a correre, a correre, in treno, in automobile, in ferrovia, in bicicletta, su i piroscafi. Correre, vivere! (Mondo di carta)

- Ajo ishte mësuar të fluturonte, të vraponte, të nxitonte me tren, me makinë, me biçikletë, me anije. Të nxitonte, të jetonte! (Botë letre, p. 66, «Zoja prej dylli», Toena, Tiranë 2008)

Nella maggior parte degli esempi soprariportati si nota che le ripetizioni dei verbi vengono date anche nelle novelle tradotte. I traduttori hanno compreso questa figura retorica usata da Pirandello e hanno avuto la saggezza di trasmetterla anche nella lingua albanese rispettando così una caratteristica del linguaggio dell'autore. Fanno eccezione gli ultimi due esempi in cui le ripetizioni non vengono riportate nella lingua albanese.

In alcuni esempi si crea la figura dell'epanalessi oppure la geminazione che consiste nel raddoppiare (geminare) un'espressione, ripetendola all'inizio, alla metà, o alla fine di un segmento testuale (Garavelli B.M., 1989: 191).

$>$ Gli sorrisi. Mi fece una nuova e più diffusa spiegazione della sua grande impresa lì su i luoghi? Gli sorrisi... Sì? davvero? Oh che piacere! Gli sorrisi. ... Sì? davvero? Oh che piacere! E gli sorrisi anche quando in cantina, 
con un'aria da Carlomagno mi mostrò quattro lunghe andane di botti... E sorrisi anche quando, più morto che vivo, rientrai nella villa... E sorrisi anche al suocero, mio ammiratore... (Le sorprese della scienza)

- I I buzëqesha. Më dha një shpjegim të ri dhe më të plotë të punës së madhe që kishte bërë në ato vende? ! buzëqesha.... Ashtu? Vërtet? Sa gjë e mirë! I buzëqesha... Ashtu? Vërtet? Sa gjë e mirë! E i buzëqesha edhe kur më pas, sikur të qe Karli i Madh, më tregoi poshtë në qilar katër radhë të gjata me fuçi... Dhe buzëqesha edhe kur, i rraskapitur dhe i dërrmuar, u ktheva në vilë... Dhe i buzëqesha edhe të vjehrrit, adhuruesit tim... (Befasitë e shkencës, p. 104, "Tregime e novela", Dituria, Tiranë 1999)

L'epanalessi è stata data anche nella novella tradotta.

\subsection{Ripetizioni di avverbi:}

$>$ Niente... niente... niente Dimissionario! dimissionario! Niente. (Niente)

- Asgië... asgië... asgië... Sikter të gjitha! Asgië! (Hiç asgjë, p. 90, «Zoja prej dylli», Toena, Tiranë 2008)

$>$ Mi spavento. Niente, niente, scusi, me ne vado. (Mondo di carta)

- Më kallni datën. Śka gië, s'ka gië, po shkoj. (Botë letre, p. 63, «Zoja prej dylli», Toena, Tiranë 2008)

$>$...arrabbiato con se stesso che non poteva ritener nulla, nulla, nulla di quanto studiava. (Scialle nero)

- ...i xhindosur me vehten e tij se nuk mbante mënd asgië, asgië, asgië nga ato që mësonte. (Shalli i zi, p. 31, «Jeta lakuriq», Naim Frashëri, Tiranë 1963)

Nei casi soprariportati gli avverbi vengono trasmessi anche nelle novelle tradotte.

> Ma sì, Dio mio, sì - tutta quella furia, sì, con ragione: chi dice di no? (Niente)

- Posi jo, o Zot i madh, patjetër që kishte të drejtë të tregonte aq ngut (Hiç asgjë, p. 78, «Zoja prej dylli», Toena, Tiranë 2008)

$>A h, \underline{\text { sì }}$ sì; per questa parte, sì̀, ha ragione... (Niente)

- Aha, s'ka dyshim, për sa i përket kësaj, keni të drejtë... (Hiç asgjë, p. 83, «Zoja prej dylli», Toena, Tiranë 2008)

Negli ultimi due esempi si nota che i traduttori non hanno ripetuto l'avverbio di affermazione sì, ma ottengono frasi affermative ricorrendo all'uso di altre parole affermative.

> No! Così no! Così no! per carità! - si mise a gridare. (Mondo di carta)

- Jo! Jo kështu! Jo kështu, pashi Zotin! - zuri të thërriste. (Botë letre, p. 64, «Zoja prej dylli», Toena, Tiranë 2008)

> Povero Gerlando! Faceva compassione, così grosso, così duro, così ispido, con un libro aperto davanti. (Scialle nero)

- I gjori Xherland! Të dhimbsej duke e parë aq të trashë, aq të pagdhendur, aq kabà, me një libër përpara, të hapur. (Shalli i zi, p. 18, «Jeta lakuriq», Naim Frashëri, Tiranë 1963)

$>$ Piano! Piano, per carità! Ma che piano!

- Ngadalë, ngadalë pashi Zotin! Kujt i thua! (Hiç asgjë, p. 85, «Zoja prej dylli», Toena, Tiranë 2008)

Nell'ultimo esempio la ripetizione non viene riproposta nella lingua albanese. avverbi.

In alcuni casi, gli elementi ripetuti accettano, intercalati, altri elementi come pronomi, frasi intere, vocativi e altri

> Ma piano con questa vettura!... [...] Ma piano con questo correre. (Le sorprese della scienza)

- Karrocë i thënçin. [...] Shpejt sa për të thënë. (Befasitë e shkencës, p. 100, "Tregime e novela", Dituria, Tiranë 1999)

Nella lingua albanese la ripetizione non viene riprodotta.

In qualche caso si produce la figura del polittoto (o poliptoto) la ripetizione di un vocabolo con funzioni sintattiche differenti nello stesso enunciato oppure negli enunciati vicini legati tra loro:

$>$ Qua sono tutti in perpetua attesa di ciò che ci porterà il domani. Qua non si fabbricano case perché domani, domani chi sa come si fabbricheranno le case; non si pensa a illuminare le strade, perché domani chi sa che nuovi mezzi d'illuminazione scoprirà la scienza, domani! E così anch'io dovrei stare in attesa del rimedio di domani... (Acqua e lì)

- Këtu të gjithë rrinë e presin ç'do të sjellë e nesërmia. Këtu nuk ndërtohen shtëpi sepse nuk dihet si kanë për t'u ndërtuar nesër, nuk mendojnë të ndriçojnë rrugët, sepse nesër s'dihet ç'mjete ndriçimi do të zbulojë shkenca, nesër! Prandaj edhe unë duhet të rri e të pres ilaçin e të nesërmes... (Ujë dhe pikë, p. 188, «Nata e parë», Toena, Tiranë 2008) 
II termine domani è poliptoto perché si usa con valori sintattici differenti. (Navarro Blanco, 1990: 120). D'altra parte qui si tratta anche di un caso di epanadiplosi (detta anche ciclo o inquadramento, una figura retorica che consiste nella ricorrenza di una o più parole all'inizio e alla fine di una frase 0 di un verso), perché abbiamo la ripetizione di una parola all'inizio e alla fine della frase.

\subsection{Ripetizioni di pronomi}

Le forme pronominali completano il loro vuoto semantico appoggiandosi in altri elementi della frase, come possono essere le preposizioni, dando luogo a esempi di figure retoriche come l'epanalessi ed il poliptoto (già precedentemente definiti).

Esempi di epanalessi:

$>$ Se qualcosa avesse fatto di male, Nelda, la signora baronessa, avrebbe scritto a me, a me, a me, non a te, per farmi un piacere! (Nel dubbio)

- Po të kishte ndodhur e kundërta, Nelda, zonja baroneshë, do të më kishte shkruar mua, mua dhe jo ty, pë të ma përplasur! (Dyshimi, p. 140, «Zoja prej dylli», Toena, Tiranë 2008)

$>$ Questo, questo cerco! Questo voglio! (II fumo)

- Këtë, këtë kërkoj! Këtë dua! (Tymi, p. 90, «Jeta lakuriq», Naim Frashëri, Tiranë 1963)

Esempi di poliptoto:

> perché le era caro far rivivere così in sé, per sé sola, il ricordo - non di lui, non di lui! - ma di tutto il male e di tutto il bene che le era venuto un giorno da lui. (La maestrina Boccarmé)

- Sepse e kishte për zemër që të rijetonte në vetvete, vetëm për veten e saj, jo kujtimin e tij, në asnjë mënyrë, por kujtimin e gjithë të keqes dhe gjithë të mirës që një ditë i kishte ardhur prej tij. (Mësuesja Bokarme, p. 135, "Tregime e novela", Dituria, Tiranë 1999)

Si nota che nelle novelle tradotte questi casi di epanalessi e poliptoto sono stati dati creando così lo stesso effetto creato sui lettori dell'originale.

\subsection{Ripetizioni di sostantivi}

$>$ lo? - esclamò Sciaramè con un balzo. - Non lo vidi? io? Ah, se lo vidi! E gli baciai anche le mani! A Piazza Pretorio, gliele baciai, a Palermo, dove s'era accampato! Le mani! (Le medaglie)

- Kush, unë? - thirri Sharameja, duke brofur përpjetë. - S'e paskam parë? Unë? E kam parë që ç'ke me të! E i kam puthur edhe duart! Në Sheshin Pretorio ia kam puthur, në Palermo, ku kishte ngrehur fushimin! Duart, po! (Medaljet, p. 24, «Zoja prej dylli», Toena, Tiranë 2008)

$>$ Dio, che rovina, che rovina, che rovina! (Come gemelle)

- Zot u shkatërrova, u shkatërrova, u shkatërrova! (Si dy binjake, p. 119, «Zoja prej dylli», Toena, Tiranë 2008)

Nell'ultimo esempio il traduttore ha riprodotto la ripetizione nella novella tradotta ricorrendo alla strategia della trasposizione, il passaggio da una parte all'altra del discorso. In questo caso si passa dal sostantivo (originale) al verbo (traduzione)

> Sì. Sì, da stupidi! da stupidi! da stupidi! (Niente)

- Punë budallenjsh! Budallenjsh që ç’ke me të. (Hiç asgjë, p. 85, «Zoja prej dylli», Toena, Tiranë 2008)

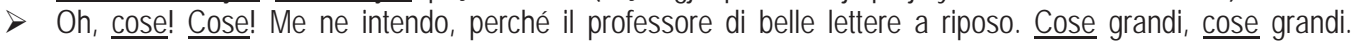
(Niente)

- Hajde vargje, hajde! Unë kam haber sepse jam profesor letërsie në pension. Alamet veprash, alamet veprash! (Hiç asgjë, p. 87, «Zoja prej dylli», Toena, Tiranë 2008).

In questi ultimi due esempi non viene riprodotta integralmente la ripetizione. Stupido si ripete tre volte nell'originale, mentre nella traduzione soltanto due volte. Lo stesso si può dire per il sostantivo cose ripetuto quattro volte nell'originale e soltanto tre nella traduzione.

$>$ Vanità... vanità... (La fede)

- Kotësi... kotësi... (Besimi, p. 52, «Zoja prej dylli», Toena, Tiranë 2008)

$>$ Che crudeltà, che crudeltà di spettacolo, quel sonno di vecchio! (La fede)

- Sa gië e pashpirt, Sa gië e pashpirt ishte ai gjumë plaku. (Besimi, p. 52, «Zoja prej dylli», Toena, Tiranë 2008)

$>$ Un pianto che gli bruciava gli occhi e lo strozzava. Pianto d'avvilimento, pianto di rabbia e di pietà insieme.... [...] Commosso e pieno di rimorso per quel pianto, don Angelino si pentì della sua durezza... (La fede) 
- Ishte ai një vaj që po i digjte sytë dhe po e mbyste. Vaj poshtërimi, vaj inati e mëshirë trazuar... [...] I prekur dhe me zemër të vrarë nga ai vaj, don Anxhelino u pendua që ishte sjellë ashpër (Besimi, p. 53-57, «Zoja prej dylli», Toena, Tiranë 2008)

$>$ A casa, a casa, voglio andare a casa! (Mondo di carta)

- Në shtëpi, dua të shkoj në shtëpi! (Botë letre, p. 60, «Zoja prej dylli», Toena, Tiranë 2008)

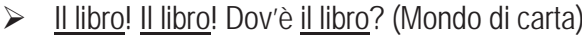

- Libri! Libri! Ku e kam librin? (Botë letre, p. 60, «Zoja prej dylli», Toena, Tiranë 2008)

$>$ Era così, e basta. II suo mondo. II suo mondo di carta. Tutto il suo mondo. (Mondo di carta)

- Ishte ashtu dhe pikë. Bota e tij. Bota e tij prej letre. E tërë bota e tij. (Botë letre, p. 67, «Zoja prej dylli», Toena, Tiranë 2008)

> Dimmi dov'è, o t'ammazzo! Sanque, sanque, voglio sanque, questa sera! Sanque! (Certi obblighi)

- Tregomë ku është, ose të vrava! Gjak, gjak, dua gjak sonte! Gjak! (Disa lloj detyrimesh, p. 164, «Zoja prej dylli», Toena, Tiranë 2008)

$>\underline{\| \text { sole }}$, il sole vero, il sole della giornata non era forse mai sorto per lui: per lui sorgevano ancora i soli del tempo di Virgilio. (II fumo)

- Dielli, dielli i vërtetë, dielli i ditëve tona, nuk kish lindur kurrë për të; për të lindnin akoma diejt e kohës së Virgjilit. (Tymi, p. 69, «Jeta lakuriq», Naim Frashëri, Tiranë 1963)

In alcuni esempi si nota una progressione dell'idea rispetto alla prima formulata come una specie di amplificazione dell'informazione contenuta nel primo enunciato, intenzione più o meno data anche nelle novelle tradotte.

\subsection{Ripetizioni di aggettivi}

$>$ Che libri! Che donne! Che casa! (Niente)

- CĆlibra, mor! Çfarë grash! Çfarë shtëpie! (Hiç asgjë, p. 90, «Zoja prej dylli», Toena, Tiranë 2008)

$>$ Carini! carini! carini! (Le sorprese della scienza)

- Sa të lezetshëm! Sa të lezetshëm! (Befasitë e shkencës, p. 104, "Tregime e novela", Dituria, Tiranë 1999)

Nel secondo esempio la ripetizione dell'aggettivo carini (tre volte) non viene resa completamente nella novella tradotta (ripetuto solo due volte).

$>$...entrò la vecchia sorella di don Piero, piccola, cerea, vestita di nero, con un fazzoletto nero di lana in capo, più curva e più tremula del fratello. Parve a don Angelino che - chiamata dalle sue lagrime - entrasse nella cameretta la sua mamma, piccola, cerea e vestita di nero come quella. (La fede)

- ...hyri brenda motra plakë e don Pjetros, një grua e vockël, si të qe prej dylli, veshur me të zeza, hedhur një shami të zezë leshi mbi krye, edhe më e përthyer nga i vëllai e që dridhej edhe më shumë nga ai. Don Anxhelinos iu duk se në dhomë, ndoshta e thirrur nga lotët e tij, kishte hyrë nëna e vet, e imët, si prej dylli, e veshur me të zeza si dhe ajo tjetra. (Besimi, p. 52, «Zoja prej dylli», Toena, Tiranë 2008)

In questo esempio l'aggettivo piccola non viene dato nella lingua albanese con una ripetizione. Le parole usate nella traduzione (e vockël - e imët) hanno lo stesso significato semantico, ma non viene creata la figura retorica.

$>\quad$ Nudo, nudo, nudo, cara mia; carissima mia, nudo! Potevo venir giù? (Nel dubbio)

- Isha çupalak, krejt çupalak, e dashur, si më ka bërë nëna! A mund të zbritja? (Dyshimi, p. 139, «Zoja prej dylli», Toena, Tiranë 2008)

In questo esempio l'aggettivo nudo ripetuto per quattro volte nella novella originale appare solamente due volte nella traduzione.

$>$ Non aveva più diritto a nessuna considerazione, a nessun compatimento. (Scialle nero)

- Nuk ish më e denjë për asnjë konsideratë, për asnjë dhëmbshuri. (Shalli i zi, p. 15, «Jeta lakuriq», Naim Frashëri, Tiranë 1963)

$>$ Bello! bello! bello! bello! bello... (II fumo)

- e bukur! e bukur! e bukur! e bukur! e bukur! (Tymi, p. 67, «Jeta lakuriq», Naim Frashëri, Tiranë 1963)

$>$ Rovinato! Rovinato! Rovinato! (II fumo)

- U shkatrrova! U shkatrrova! U shkatrrova! (Tymi, p. 81, «Jeta lakuriq», Naim Frashëri, Tiranë 1963)

$>$ Ottenuta la laurea, s'eran subito buttati al lavoro, come due bestie; con tanto impegno, con tanto accanimento che in poco tempo erano riusciti a bastare a se stessi. (Scialle nero)

- Me të marrë diplomat u dhanë shpejt pas punës, si dy kafshë; duke punuar pareshtur e me vrull, në një kohë të shkurtër u bënë të zotët e vetes dhe u mëkëmbën. (Shalli i zi, p. 15, «Jeta lakuriq», Naim Frashëri, Tiranë 
1963)

$>\quad$...quegli urli, quei fischi, quelle minacce del popolo... (Visto che non piove)

- ...me të thirrura, fishkëllima dhe këcënime të popullit... (Meqë s'po bie shi, p. 51, «Nata e parë», Toena, Tiranë 2008)

Negli ultimi esempi riportati le ripetizioni dell'aggettivo quantitativo tanto e dell'aggettivo dimostrativo quello non vengono date nella novella tradotta.

\subsection{Ripetizioni di altri elementi}

$>$ senza vie, senz'acqua, senza luce... (Le sorprese della scienza)

- pa rrugë, pa ujë, pa drita...(Mësuesja Bokarme, p. 102, "Tregime e novela", Dituria, Tiranë 1999)

$>$ Ed allora essa, un po' per pietà, un po' per ridere, un po' per darsi da fare, s'era messa ad ajutare quel povero giovanotto, fin dove poteva. (Scialle nero)

- Atëherë ajo, ca për mëshirë, ca për t'u argëtuar, ca për t'u englendisur e për t'u marrë me një punë, kish nisur ta ndihmojë atë djalosh fatkeq, me sa mundesh. (Shalli i zi, p. 19, «Jeta lakuriq», Naim Frashëri, Tiranë 1963)

$>$... che la smettesse con questa scuola, con questa scuola, con questa scuola! Non ne poteva più! (Scialle nero)

- ...të mos e torturonte më me atë shkollë, me atë shkollë, me atë shkollë! Nuk mund të duronte më! (Shalli i zi, p. 18, «Jeta lakuriq», Naim Frashëri, Tiranë 1963)

In quasi tutti i casi soprariportati si tratta di elementi che si ripetono per ben tre volte, caratteristica che viene data anche nella traduzione.

Procedimento anaforico è inoltre il polisindeto, oppure la coordinazione tramite le congiunzioni. Facilmente riconoscibile in qualsiasi tipo di comunicazione linguistica, nella lingua letteraria la sua frequenza può definire lo stile di uno scrittore o di un'opera. In questo senso bisogna dire che i racconti di Luigi Pirandello si presentano come una lunga serie di catene anaforiche polisindetiche (Navarro Blanco, 1990: 124).

Esempi di polisindeto nelle frasi:

$>\underline{E}$ fischi $\underline{e}$ urli $\underline{e}$ invettive sotto il palazzo vescovile... (Visto che non piove)

- Dhe ia nisnin të sharat dhe fishkëllimat aty rrëzë pallatit të Ipeshkvisë... (Meqë s'po bie shi, p. 50, «Nata e parë», Toena, Tiranë 2008)

> ...belli $\underline{e}$ ballanti $\underline{\underline{e}}$ sonanti erano i denari della dote che il Vescovo era stato costretto a dargli... (Visto che non piove)

- ...fort të bukura e tingëlluese ishin parickat dhe paja që u detyrua t'i jepte imzot Ipeshkvi; (Meqë s'po bie shi, p. 51, «Nata e parë», Toena, Tiranë 2008)

$>$ Non è vero che legge! Mi ci vien sopra! $\underline{0}$ che non veda, $\underline{0}$ che vada stordito, $\underline{0}$ che $\underline{0}$ come, fatto si è... (Mondo di carta)

- Nuk është e vërtetë që lexon! Gati sa s'më shkel me këmbë! Ose nuk sheh ose ecën kuturu, një dreq e di, por puna është se... (Botë letre, p. 59, «Zoja prej dylli», Toena, Tiranë 2008)

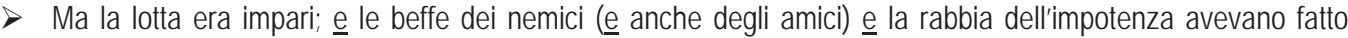
perdere a Spatolino il lume degli occhi. (II tabernacolo)

- Por lufta ishte e pabarabartë dhe talljet e armiqve (ashtu sikundër edhe të miqve) bashkë me mërinë që i ngjallte pafuqia, e kishin tërbuar fare Spatolinon. (Tabernakulli, p. 78, «Nata e parë», Toena, Tiranë 2008)

$>$...pensavano che questi, $\underline{0}$ per incarico del vescovo $\underline{0}$ per voto della Società Cattolica, costruisse lì quel tabernacolo, per far dispetto al vecchio usurajo. (II tabernacolo)

- ...mendonin se ai, me porosi të Ipeshkvit dhe me dëshirën e Shoqatës Katolike, po e ndërtonte shi aty atë tabernakull për inat të fajdexhiut plak. (Tabernakulli, p. 85, «Nata e parë», Toena, Tiranë 2008)

$>$...tutti ne avrebbero sofferto; $\underline{e}$ allora, cominciava la distruzione, altre bocche d'inferno si sarebbero aperte $\underline{\mathrm{e}}$, in pochi anni, tutti gli alberi, tutte le piante sarebbero morti, attossicati dal fumo, e addio campagne! (Fumo)

- Do vuanin gjithë të tjerët. Atëherë, do të fillonte shkatërrimi, të tjera gojë do të hapeshin dhe, në pak vjete, të gjitha pemët, të gjitha bimët do të vdisnin, të helmuara nga tymi, dhe: lamtumirë fusha! (Tymi, p. 54, «Jeta lakuriq», Naim Frashëri, Tiranë 1963)

Negli esempi soprariportati si nota che questi esempi di polisindeto non vengono dati nelle traduzioni. La sintassi albanese non richiede l'uso di tante congiunzioni nello stesso periodo come avviene invece nelle novelle di Pirandello. $\mathrm{E}$ di fronte a questa caratteristica del linguaggio dello scrittore i traduttori hanno pensato bene a rispettare la sintassi della 
Ioro propria lingua non rispettando però la caratteristica della lingua dello scrittore.

Esempi di reiterazione possiamo inoltre considerare anche quelle formule nelle quali la ripetizione si ottiene tramite una parola della stessa radice della prima, quasi sempre con qualche prefisso. Costituiscono chiari esempi di figure etimologiche nelle quali entra in gioco la derivazione delle parole.

$>\quad$ (...) lo guarda sbigottito e cerca di confortarlo. Conforti che, inutile darli, eppure si danno. (Acqua e lì)

- E vështron i shastisur dhe përpiqet ta ngushëllojë. Ngushëllime që janë krejt të kota të bëhen, por që bëhen njëlloj. (Ujë dhe pikë, p. 188, «Nata e parë», Toena, Tiranë 2008)

In questo esempio si ottiene una combinazione di una figura etimologica ed una anadiplosi visto che l'ultima parte di una frase (confortarlo), appare nella prima parte della frase successiva (conforti) (Navarro Blanco, 1990: 126). Anche nella traduzione albanese questi casi di reiterazioni sono facilmente intuibili da parte dei lettori dato che i traduttori li hanno trasmessi.

Altri esempi:

> Io la ho avvertita! Bell'avvertimento! (Mondo di carta)

- Unë po ju paralajmëroj! Lëre mos e pyet ç'paralajmërim! (Botë letre, p. 61, «Zoja prej dylli», Toena, Tiranë 2008)

$>$ Ma se è tutto finito, strafinito, arcifinito! Ancora? (Nel dubbio)

- Po ajo punë ka mbaruar, ka stërmbaruar, stër-stër-stërmbaruar! Prapë ai avaz? (Dyshimi, p. 140, «Zoja prej dylli», Toena, Tiranë 2008)

$>$ Si sentiva forse vincolato da questo sacrifizio per tutta la vita, condannato a sacrificare a sua volta la propria gioventù, la libertà dei proprii sentimenti alla sorella? (Scialle nero)

- Për hir të asaj sakrifice mos ndjehej vallë i ndrydhur e i detyruar për tërë jetën, i dënuar të sakrifikojë edhe ay lirinë e tij, lirinë dhe ndjenjat e pasionet, për të motrën? (Shalli i zi, p. 15, «Jeta lakuriq», Naim Frashëri, Tiranë 1963)

$>$ don Camillo inciampò in un fantoccio da sarta; all'inciampone (...) (Come gemelle)

- don Kamilo u pengua në një manekin kartuçi nga ata që përdorin rrobaqepësit; kur hasi në të (...) (Si dy binjake, p. 119, «Zoja prej dylli», Toena, Tiranë 2008)

Come abbiamo precedentemete detto è evidente la presenza della derivazione in tutti i casi di reiterazione cosa che nella maggioranza dei casi viene data anche nelle novelle tradotte in albanese ad eccezione di quest'ultimo esempio in cui la reiterazione non viene riprodotta.

\section{Conclusioni}

In questo studio abbiamo preso in analisi una caratteristica del linguaggio delle novelle di Pirandello, le ripetizioni, questa figura retorica tanto usata dallo scrittore nella sua prosa e che caratterizza il suo stile molto vicino alla lingua parlata. Abbiamo notato che, generalmente, i traduttori hanno trasmesso questa figura anche nelle novelle tradotte in albanese, ad eccezione di alcuni casi in cui i traduttori non riproducono nei testi tradotti le ripetizioni che Pirandello mette in bocca dei suoi personaggi. Per concludere dobbiamo dire che, trattandosi di una peculiarità dello stile dell'autore occorre dare questa figura retorica anche nella lingua d'arrivo per rispettare così intentio-auctoris e per creare sul lettore della traduzione lo stesso effetto prodotto anche sul lettore dell'originale.

\section{References}

\section{Fonti Primarie}

Borzi, I. \& Argenziano, M. (1993). Luigi Pirandello Novelle per un anno, Roma: Newton\&Compton Editori.

Pirandello, L. (1999). Tregime e Novela, Tirana: Dituria. , (2008). Nata e parë, Tirana: Toena. (2008). Zoja prej dylli, Tirana: Toena. (1997). Novela për një vit. Jeta lakuriq, Tirana: Çabej. (2010). Teze Mikelina, Tirana: Toena.

\section{Fonti Secondarie}

Bally, Ch. (1951). Traité de stylistiques français, Geneva.

Carbonell i Cortés O., (1999). Traducción y cultura: de la ideología al texto. Madrid: Ediciones Colegio de España. 
Catford, J. C, (1965). A Linguistic Theory of Translation, An Essay in Applied Linguistics, Language and language learning. London: Oxford University Press.

Garavelli, B.M. (1989). Manuale di retorica, Milano: Bompiani.

Kartsevski, S. (1941). Introduction à l'étude de l'interjection, in "Cahiers de F. de Saussure", N. 1.

Kostallari, A. (1980). Fjalor i gjuhës së sotme shqipe, Tirana: Naim Frashëri.

Lefevere, A. (1992). Translating Literature: Practice and Theory in a Comparative Literature Context. New York: The Modern Language Association of America.

Leka, F. \& Simoni, Z. (2000). Fjalor italisht-shqip, Tirana: Infbotues.

Munafò, G. (1982). Conoscere Pirandello, Firenze: Le Monnier

Navarro Blanco, A. (1990). La lengua y el estilo de Luigi Pirandello, tesis doctoral.

Papa, M. (2011). Sulla traduzione nella lingua albanese delle opere del nobelista italiano Luigi Pirandello, tesi dottorale.

Puglisi, F. (1968). Pirandello e la sua lingua, Bologna: Cappelli editore.

Rabadán, R. (1994). Traducción, intertextualitad y manipulación. in Amparo Hurtado Albir (ed.) Estudis Sobre la traducció. Castelló: Universitat Jaume I, 129-39.

Spitzer, L. (1922). Italienische Umgangssprache, Bonn-Leipzig.

Terracini, B. (1982). Analisi Stilistica, Teoria, storia e problemi, Milano: Feltrinelli.

Terracini, B. Considerazioni sullo stile delle novelle di Pirandello, në Atti del Congresso Internazionale di Studi Pirandelliani, f. $729-734$.

Zingarelli, N. (2002). Vocabolario della lingua italiana, Bologna: Zanichelli. 